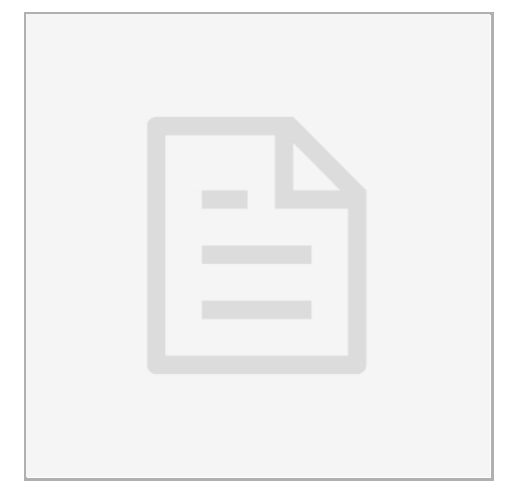

MAY 08, 2020

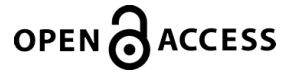

DOI:

dx.doi.org/10.17504/protocol s.io.bf2ijqce

Protocol Citation: Michelle Daniel, Marda Jorgensen 2020. Imaging Mass Cytometry Data Acquisition. protocols.io

https://dx.doi.org/10.17504/p rotocols. io. bf 2 ijqce

License: This is an open access protocol distributed under the terms of the Creative Commons Attribution License, which permits unrestricted use, distribution, and reproduction in any medium, provided the original author and source are credited

Protocol status: Working We use this protocol and it's working

Created: May 05, 2020

Last Modified: Oct 02, 2020

PROTOCOL integer ID: 36650

Keywords: HuBMAP, TMCFlorida/Zurich, Imaging Mass Cytometry

\section{(3) Imaging Mass Cytometry Data Acquisition}

\author{
Michelle Daniel $^{1}, \quad$ Marda Jorgensen ${ }^{2}$ \\ ${ }^{1}$ University of Zürich; ${ }^{2}$ University of Florida

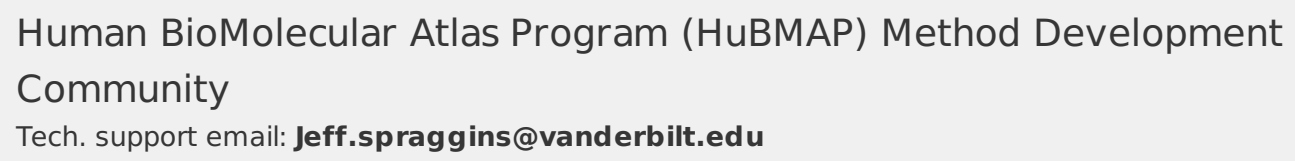

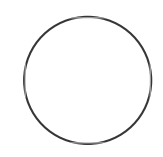

Marda Jorgensen

\section{ABSTRACT}

This SOP describes the image acquisition with an Imaging Mass Cytometer (IMC or Hyperion).

Prior to this protocol the IMC needs to be fully tuned to specifications (see "HuBMAP_Protocol_4_IMC_Tuning.pdf”). Additionally, every batch of samples stained

with the same antibody-mix requires the acquisition of a compensation slide for spill over

correction (see "HuBMAP_Protocol_3_Compensation_Slide_Preparation.pdf" \& "HuBMAP_Protocol_6_IMC_Compensation_Slide_Acquisition.pdf”).

\section{GUIDELINES}

IMC: Imaging Mass Cytometer or Hyperion (Commercial name by manufacturer).

FFPE: Formalin-fixed, paraffin-embedded.

Slide: Refers to a microscopy glass slide containing one or multiple Samples.

Sample: Refers to either a clinical FFPE tissue section from a patient or a cell pellet cytoblock FFPE control sample on a glass slide.

Unstained sample: Refers to a sample prior to antibody staining.

Stained sample: Refers to a sample after antibody staining.

ROI: Region of interest

\section{MATERIALS}

\section{MATERIALS}

\section{IMC (software 7.0 and higher) Fluidigm}

88 Flat-bed scanner with custom sample holder Contributed by users

$\$ 8$ Slide containing stained samples Contributed by users 


\section{Set up the flat-bed scanner}

1 1. Attach the flat-bed scanner to a computer.

\section{Create overview images of the slides}

2 1. Use the flat-bed scanner with the custom sample holder and load up to 12 slides to be acquired.

2. Scan the slides to .jpg at a resolution of $600 \mathrm{dpi}$ and save the image.

Note

The low resolution overview scanning of each slide can be performed on any computer.

\section{MCD ffle setup and Panorama creation}

3 1. Insert slide into the IMC.

2. Under "Data acquisition" create a new Empty12AF file.

3. Make sure that the autofocus pins in the software are not located above the hydrophobic marker

pen. This may lead to the creation of a false focal plane image of the slide and result in decreased ablation efficiency.

4. Save the mcd file in the data drive ("E") as a new folder.

5. Create panorama images of the tissue samples. Note: Panoramas should not be larger than $10^{\prime} 000 \times 10^{\prime} 000$ pixels.

Note

This requires a running, fully tuned IMC.

\section{ROI selection}

4 1. Locate the areas of interest identified in IHC staining in the panorama.

2. Set up an ROI for the region(s) of interest with a defined area(s) (e.g. $1000 \times 1000$ pixels).

3. In AirLab download the relevant panel (in options select "for CyTOF 2"). Save it to the folder 
where the mcd file is located.

4. Select all ROIs and under "Template" import the panel downloaded from AirLab.Scroll to the bottom of the list and select the imported panel. In the panel selection pane, set the ablation energy to $2.5 \mathrm{~dB}$ and the Ablation Frequency to $400 \mathrm{~Hz}$.

5. Double check that the right template was selected and that Ir191 and Ir193 are included.

6. Hit select template.

7. Set the DV Opt parameter to $4 \mathrm{~h}$.

8. Check the "Generate Text File" box.

9. Hit start. (1 mm2 at $400 \mathrm{~Hz}$ should roughly take $1 \mathrm{~h}$ ).

Note

Once the panel has been downloaded from AirLab and added to the first acquisition it is available in the IMC software. It will then not be necessary to repeat this step afterwards and it will also

not be necessary to save the panel.conf to every single folder that is created for each slideID. 